\title{
GÉNERO Y VOCACIÓN CIENTÍFICA Un estudio de caso basado en mecanismos
}

\author{
GENDER AND SCIENTIFIC VOCATION \\ A Mechanisms-based Case Study
}

\author{
FRANCISCO José LEÓN \\ Universitat Autònoma de Barcelona. España \\ franciscojose.leon@uab.cat \\ EnRICO MORA \\ Universitat Autònoma de Barcelona. España \\ enrico.mora@uab.es
}

\begin{abstract}
RESUMEN
A pesar de los avances hacia la igualdad, la plantilla del personal académico de las universidades españolas continua siendo fundamentalmente masculina. De los distintos filtros que actúan impidiendo el progreso académico de las mujeres hacia categorías superiores, aquellos que tienen lugar en el periodo de solicitud y disfrute de las becas de investigación son especialmente importantes. En ese periodo tiene lugar un proceso diferencial de construcción de subjetividades para mujeres y hombres cuyo efecto principal es una elaboración diferencial de las expectativas y preferencias profesionales para el futuro. Basándonos en el análisis de 40 entrevistas ( 18 realizadas al personal becario y 22 al profesorado de la Universidad Autónoma de BarceIona) y de una encuesta online realizada a 258 becarios/as de la UAB, tratamos de identificar algunos mecanismos causales que pueden ayudarnos a explicar por qué se produce una diferencia en las probabilidades de formación de un orden de preferencias que sitúe a la actividad científica como objetivo profesional prioritario, es decir, por qué los hombres desarrollan más frecuentemente que las mujeres una vocación científica.
\end{abstract}

\section{Palabras Clave}

Carrera académica, Ethos científico, Personal becario, Sexo.

\section{Abstract}

Despite the advances towards equality, the academic staff of the Spanish universities is still predominant by masculine. From the different filters that are avoiding the academic progress of women towards superior categories, those that take place in the application and enjoyment of research grants are especially important. In that period, it is taking place a differential process of subjectivities construction for women and men, the principal effect of which is a differential elaboration of professional expectations and preferences for the future. From the analysis of 40 interviews (18 conducted with grant staff and 22 with academic staff of the Autonomous University of Barcelona), and an online survey made to 258 scholarship holders of the AUB, we try to 
identify some causal mechanisms that help us to explain why is there a difference in the probability of forming of a preference order that places the scientific activities as a priority professional goal, that is to say, why men develop a scientific vocation more often than women.

\section{KEWORDS}

Academic Career, Grant staff, Scientific Ethos, Sex.

\section{INTRODUCCIÓN}

En contra de lo que pudiera parecer, la Universidad, como espacio de trabajo, es un lugar más sexista que el mercado de trabajo, al menos en lo que se refiere al personal docente (Izquierdo et al. 2004). La desigualdad en la proporción de mujeres y hombres es mayor cuanto más alta es la categoría del profesorado, alcanzando en sus categorías altas niveles de desigualdad superiores a los existentes entre el personal directivo de las empresas privadas. Como ya se ha indicado en estudios anteriores (Izquierdo et al. 2004), no parece que el concepto de "techo de cristal" capture adecuadamente este fenómeno. En el especial escenario de la vida académica, la imposibilidad de progresar no se traduce en un estancamiento, sino en una inevitable retirada de la actividad científica universitaria. En este artículo expondremos algunos de los principales resultados de un trabajo de investigación que ha pretendido arrojar algo de luz sobre este fenómeno.

Los estudios sobre mujeres y ciencia han solido centrarse en el análisis de la demanda, poniendo el énfasis en los procesos de discriminación, segregación, etc. Nuestro enfoque se centrará en la oferta, en concreto, en los modos en que la actividad científica predoctoral va gestando una construcción diferencial de preferencias profesionales entre mujeres y hombres. Esta diferencia puede capturarse mediante el concepto de vocación, entendiendo por ella una preferencia marcada y poco flexible por la actividad científica. Nuestro análisis tratará de identificar mecanismos que expliquen el vínculo causal entre las condiciones sociales en las que se desarrolla la actividad predoctoral, y las preferencias manifestadas respecto de la vida profesional futura. Para ello, nos centraremos en el análisis de un caso: el del personal becario de la Universidad Autónoma de Barcelona (UAB, en adelante). El análisis se basa en el material aportado por 40 entrevistas realizadas a personal becario y profesorado de la UAB, y por una encuesta online realizada a 258 becarios/as de la UAB. Los resultados que exponemos forman parte de la investigación Diagnosis de la trayectoria académica del personal becario de la UAB desde la perspectiva de género, dirigida por Enrico Mora en el marco del Observatori per a la lgualtat de la UAB y del Grup d'Estudis Sentiments, Emocions i Societat (GESES) de la misma Universidad, y que contó con la financiación de la AGAUR (Generalitat de Catalunya).

La tesis fundamental que defenderemos será que existe realmente una diferencia en las preferencias profesionales de becarias y becarios, que tal diferencia explica en 
parte la diferente proporción en la que se estabilizan en la academia, y que ella misma es explicable como resultado de algunos mecanismos sociales.

La organización de este artículo es la siguiente: En primer lugar, planteamos el problema de la progresiva desaparición de las mujeres conforme avanzamos en la carrera académica, y acotamos nuestro interés al estudio del periodo de disfrute de los distintos tipos de becas predoctorales. A continuación, presentamos el contexto empírico general de la carrera científica en España, y repasamos la bibliografía que ha abordado el estudio de la desigualdad de género entre el personal becario. En tercer lugar, hacemos explícita nuestra apuesta por la estrategia explicativa basada en la identificación de mecanismos. En cuarto lugar, definimos el concepto de vocación y especificamos su vinculación con los principios que forman parte del ethos científico. En el quinto apartado presentamos brevemente el contexto empírico del caso que analizamos. En el sexto apartado especificamos la metodología empleada. $Y$, en séptimo lugar, analizamos e interpretamos el caso del personal becario de la UAB, tratando de especificar los mecanismos que pueden estar operando en la construcción diferencial de preferencias profesionales entre mujeres y hombres.

\section{Objeto de estudio}

\section{Planteamiento del problema}

El fenómeno de la progresiva desaparición de las mujeres de la actividad científica conforme nos movemos a categorías profesionales superiores, resulta de una combinación de factores (Clark 2005), que no tienen por qué ser los mismos en cada uno de los puntos críticos de la trayectoria académica. En este artículo, identificamos algunas variables explicativas que pueden estar contribuyendo a generar diferencias entre mujeres y hombres en un momento especialmente importante de la trayectoria: el disfrute de las becas predoctorales y de apoyo a la investigación. El período comprendido entre la licenciatura y el doctorado es un período clave en la trayectoria académica. Es entonces cuando comienzan a delinearse las preferencias y los intereses profesionales de aquellas personas que en un principio se han mostrado atraídas por la labor científica. Nuestro objetivo es identificar factores causales que contribuyan a explicar por qué se producen diferencias en función del sexo en ese proceso de construcción de creencias, preferencias e intereses. Para llevar a cabo este análisis nos apoyamos en un estudio de caso: el personal becario de la UAB. Al tratarse de un estudio de caso, los resultados no son extrapolables sin más al conjunto del personal becario de las universidades y centros de investigación españoles. La validez de los datos queda pues circunscrito a la UAB.

La elección de esta Universidad, responde, en primer lugar al cumplimiento de la Medida 2.4.1: "Llevar a cabo un estudio monográfico sobre las condiciones de trabajo del colectivo de becarios y becarias por sexo y grupo del Primer Plan de acción para la igualdad entre mujeres y hombres de la UAB, bienio 2006-2007". El plan fue aprobado 
por el Consejo de Gobierno de la UAB el 04/05/2006. Acuerdo 18/2006. Esta prioridad política nos ha permitido acceder a un conjunto de datos sobre el personal becario recopilados por la propia universidad. En segundo lugar, la UAB es uno de los principales centros universitarios docentes y de investigación de España, con presencia significativa en todos los campos del saber, lo que nos ha permitido aproximarnos empíricamente al personal becario sin omitir ningún área de conocimiento.

\section{MARCO DE ANÁLISIS}

\section{Estado de la cuestión}

En las últimas décadas del siglo XX se han producido algunos cambios importantes en la organización de las ciencias. Podemos destacar, siguiendo a Fernández Esquinas (2002), dos: por un lado, la reestructuración de las organizaciones científicas tradicionales, especialmente la universidad y, por el otro, la creciente actuación racionalizadora del Estado a través de los mecanismos de la política científica. El efecto de estos procesos en la formación del personal investigador se ha traducido, para el contexto español, en la configuración de dos modelos. El primer modelo se refiere a la formación autónoma en torno a un programa de actividades que corresponde al modelo de ciencia tradicional, y el segundo, a la formación integrada en la práctica profesional que corresponde a la ciencia colectiva.

En este marco, las becas son un tramo de la carrera académica fundamental en la política científica española de las últimas décadas, siendo la principal puerta de acceso a la práctica profesional de la ciencia en el sistema universitario español. A pesar de su importancia, la etapa becaria de la trayectoria académica ha sido poco estudiada en el contexto de las universidades catalanas y españolas, y aún menos desde una perspectiva de género. Entre las investigaciones disponibles más relevantes tenemos que referirnos al estudio de González et al. (1995) sobre la inserción laboral y las trayectorias profesionales de las becarias y becarios del Estado español (del periodo comprendido entre 1982 y 1992). Cabe destacar que la variable sexo no forma parte de los factores explicativos tomados como principales en la explicación de las diferentes carreras profesionales.

La investigación de Fernández Esquinas (2002) sobre la formación de personal investigador científico en España sitúa el análisis de la trayectoria profesional del personal becario en el contexto más amplio de las políticas de formación de personal investigador a través del Plan Nacional de I+D. Una vez caracterizado este Plan, analiza en detalle el acceso y la socialización en la ciencia académica a través de la formación predoctoral en los centros públicos universitarios y en las empresas. La variable sexo es una variable más en el análisis llevado a cabo.

En Cruz-Castro et al. (2006) se analizan las trayectorias profesionales de las investigadoras e investigadores pero atendiendo únicamente al personal del sistema público 
y considerando el impacto que sobre éste han ejercido las políticas de formación del personal investigador. Al igual que en el estudio anterior, la variable sexo aparece como una más y no es considerada como un aspecto central. Por contra, Fox (1995) se centra en la carrera académica de las mujeres científicas y su situación durante los estudios de doctorado. Observa que si bien en los últimos años ha incrementado la proporción de mujeres que hacen el doctorado, este hecho no ha supuesto el aumento de la presencia de las mujeres en las categorías más altas del profesorado. En trabajos posteriores con Paula Stephan (Fox y Stephan 2004), aborda la correspondencia entre las expectativas profesionales de los estudiantes de doctorado y la ocupación que finalmente acaban obteniendo, distinguiendo por ámbito de conocimiento y sexo.

Mary Wyer (1997) también se ocupa de la situación de las estudiantes de doctorado en la universidad pero considerando sólo los programas vinculados con las ciencias, las matemáticas y las ingenierías. A partir del estudio de tres cohortes (1985 al 1987), observa que la infrarrepresentación de las mujeres en las diversas posiciones en la carrera académica puede ser explicada por las barreras estructurales que las mujeres se encuentran al intentar avanzar en la carrera académica una vez finalizado el doctorado.

\section{Estrategia explicativa}

Los mecanismos sociales y el enfoque centrado en la oferta

La progresiva desaparición de las mujeres en la carrera académica es una regularidad estadística bien documentada en la literatura. Cuando se han tratado de identificar sus causas, los esfuerzos se han orientado tradicionalmente a la identificación de los factores "externos" a la mujer, es decir, aquellos que dan forma a la demanda: discriminación, sesgos en los procesos evaluativos (European Commission 2004), etc. Como han señalado Browne y England (1997), los enfoques centrados en la oferta han sido denostados como políticamente interesados, pues se ha entendido que culpabilizaban a las víctimas. Sin embargo, una explicación completa del fenómeno requiere también una comprensión de los procesos mediante los cuales las condiciones estructurales limitan y dan forma a las subjetividades (y así a la "oferta") teniendo éstas entonces un efecto reproductor o transformador de aquellas condiciones.

Un estrategia de explicación que trata de ir más allá de la presentación de regularidades estadísticas y que puede ayudar en la comprensión de los factores que afectan a la oferta es la estrategia de explicación basada en mecanismos (Elster 1989; 2007; Hedstrom y Swedberg 1996; 1998 y Boudon 1998; 2004). En primer lugar, esta estrategia nos aporta conocimiento sobre los procesos causales que generan los fenómenos sociales, evitando las cajas negras en la explicación (Boudon 1998), y arrojando luz así sobre fenómenos que hasta el momento permanecían opacos (Boudon 2004). Y en segundo lugar, nos proporciona mayor capacidad para diseñar medidas de intervención política efectivas (Elster 1989), al evidenciarnos las "tuercas y tornillos" que hacen moverse a la maquinaria, en este caso la maquinaria de la desigualdad. 
De la diversidad de mecanismos sociales existentes, aquí nos centraremos en lo que Hedstrom y Swedberg han llamado "mecanismos situacionales" (1996; 1998). Estos mecanismos dan cuenta de la transición macro-micro, es decir, se centran en explicar las causas de los procesos de formación de determinados estados mentales (creencias, preferencias, etc.) en un contexto estructural o situacional dado. Lo que nos interesa, por tanto, es identificar posibles mecanismos situacionales que nos ayuden a explicar el proceso de construcción de la vocación científica, tal cual la definimos a continuación.

\section{La vocación científica como producto}

\section{La vocación: definición e implicaciones prácticas}

Los miembros de la comunidad científica se caracterizan, en término medio, por una considerable implicación emocional con su tarea (Eiduson 1962; McClelland 1979; Roe 1953). Sobre la base de esa implicación, se desarrollan largas jornadas de trabajo, y en realidad, se difuminan las fronteras entre obligación y placer. Además, la comunidad científica suele representarse como una comunidad caracterizada por una exigente ética del trabajo y una entrega considerable a la profesión ${ }^{1}$. Esta constelación de creencias, preferencias y éticas del trabajo suele condensarse bajo la idea de la vocación científica.

El término "vocación" está muy presente en nuestro lenguaje cotidiano, pero estamos lejos de disponer de una definición clara, precisa y operativa del concepto. Obviamente, lo primero que es posible decir acerca de la vocación es que se trata de un estado mental, y que como tal, no es comprensible si no es considerado conjuntamente con las acciones en las que se apoya y a las que da lugar. En segundo lugar, nuestras intuiciones y prenociones sobre lo que es la vocación apuntan a algún tipo de inclinación o tendencia a vincularse estrecha y rígidamente con algún patrón definido de conducta, especialmente profesional. Así, por ejemplo, cuando decimos de alguien que tiene vocación de sacerdote, no nos referimos únicamente a que quiere dedicarse a esa profesión, sino que además (a) lo desea con una intensidad destacable, (b) ese deseo parece rígido y poco negociable, en el sentido de que cualquier otra alternativa es mucho menos deseada, y (c) el deseo apunta a un tipo de desempeño de la labor realizado desde un universo simbólico particular y dando lugar a un especial estilo de vida. Un último rasgo de la vocación es que se experimenta como resultado de procesos no-sociales. En unos casos, se presenta como resultado de una llamada "divina", y en otros, como una predeterminación inscrita en nuestra naturaleza. Uno ha sido "llamado" al sacerdocio, o "ha nacido" para ser médico. Las referencias a lo sagrado y a lo biológico presentan la vocación como destino, y así, como algo superior al individuo que la experimenta, que por tanto no se enfrenta a ella como el producto de su biografía.

\footnotetext{
${ }^{1}$ Obviamente, este tipo de vínculos requiere algún tipo de externalización del cuidado propio y de los tuyos, por lo que históricamente ha sido una posibilidad manifiestamente masculina.
} 
A partir de estas nociones, definiremos aquí la vocación científica como un ordenamiento rígido de preferencias vitales y profesionales en el que la dedicación a la actividad científica se ha situado en primer lugar, a distancia considerable de sus alternativas.

Este ordenamiento de preferencias tiene implicaciones en términos mentales y comportamentales. En primer lugar, se experimenta la vinculación con la actividad con una intensidad considerable, principalmente porque se experimenta como algo valioso y placentero en sí mismo, lo que implica un efecto feedback sobre el deseo de realizar la actividad, reforzándolo.

En segundo lugar, la posibilidad de desplegar la propia actividad en sectores ajenos al de la ciencia va difuminándose poco a poco. Normalmente, la persona entregada a la ciencia no siente el deseo de explorar en el futuro nuevas profesiones no relacionadas con el conocimiento.

En tercer lugar, sobre la base de este ordenamiento de preferencias, se difuminan las fronteras entre trabajo y ocio. Algunas actividades propias de la profesión suponen una fuente de placer en sí mismas, y por ello pasan a tener un importante papel en el tiempo libre.

En cuarto lugar, y como resultado de lo anterior, la vocación se traduce en un estilo de vida y un universo simbólico particular, que vendría a incluir todos aquellos valores, opiniones y concepciones que podamos considerar propios de la comunidad científica. La socialización en la institución científica supone una adhesión al ethos científico, sea el que sea en cada momento y lugar. Tal adhesión hace posible la integración en la comunidad científica, pues inscribe fuertemente en la personalidad del individuo las reglas de comportamiento que permiten cumplir las expectativas.

Obviamente, el modo en que se concretan en la práctica estas implicaciones es muy dependiente de las características de la comunidad o subcomunidad científica en la que se desempeña la propia labor.

La vocación científica y el ethos de la ciencia

Entender la ciencia como una institución social (Fernández Esquinas y Torres 2009) implica poner de relieve el sistema de valores y normas que regula y da sentido a las acciones que se desarrollan en su seno. Los principios fundamentales de este ethos científico fueron establecidos por Merton y desarrollados y criticados en debates posteriores -ver, por ejemplo, las aportaciones recientes de Barnes (2007), Huff (2007), Turner (2007) - . Merton (1977a) consideraba que los cuatro elementos que constituían el sistema de valores y normas propio de la institución científica eran el universalismo, el comunismo, el desinterés y el escepticismo organizado —-más adelante incluiría también la humildad y la originalidad (1977b). Estos principios contienen una dimensión moral y una técnica (Kalleberg 2007), y su función es la de regular el modo en que la institución logra su objetivo.

El planteamiento de Merton fue posteriormente criticado por autores como Barnes y Dolby (1970) y Rothman (1972), que señalaron la necesidad de distinguir entre las normas 
y la realidad empírica de las acciones que se esperaban reguladas por la norma. En esta línea, la aportación más destacada fue la de Mitroff (1974), que identificó la vigencia de una serie de contranormas, que se opondrían a las descritas por Merton, y que no necesariamente eran disfuncionales para el desarrollo del conocimiento científico. Estas contranormas serían el particularismo, el secretismo, la actitud interesada y el dogmatismo organizado. Diversos estudios se han centrado en el efecto discriminador de estas contranormas contra las mujeres y otras minorías - por ejemplo, Scott y Fox (1995).

En realidad, las cuatro normas y contranormas regulan moral y técnicamente el proceso de producción del conocimiento, pero ninguna apunta directamente al papel que la actividad científica debe jugar en la vida cotidiana del científico, que es a lo que apunta directamente la noción de vocación. Sin embargo, parece evidente que alguno de estos principios tiene implicaciones claras sobre ese papel. En concreto, el principio del universalismo apunta que la entrada y el progreso en la comunidad científica no deben resultar de atributos personales o sociales, sino únicamente del valor de las pruebas y los argumentos aportados. Este principio conduce, por tanto, a la defensa de la meritocracia como sistema deseable de acceso y promoción. En ese contexto, la ética de entrega al trabajo, que constituye uno de los ejes vertebradores de la vocación, adquiere una predominancia clara en la ubicación de la actividad científica en el conjunto de la vida cotidiana. El alcance real de esta implicación del principio de universalismo es algo a determinar empíricamente, pero hay dos hechos que parecen claros: que son raras las veces que se acepta públicamente un criterio distinto al meritocrático, y que, como mínimo a un nivel informal, la entrega incondicional al trabajo suele estar sancionada positivamente.

Por su parte, los principios del desinterés y el comunismo apuntan al reconocimiento de los pares como objetivo y recompensa fundamental de la actividad científica. Los cambios recientes en la institución científica hacen muy cuestionable que estos principios normativos regulen efectivamente, $y$ de manera general, las acciones del personal científico. El nuevo modo de producción de la ciencia apunta a una mayor interacción (y en algunos casos dependencia) de las organizaciones científicas respecto de las políticas y económicas. A este modelo se le ha llamado ciencia "post-académica" (Ziman 1996) o "post-normal" (Funtowicz y Ravetz 1993), ciencia "modo 2" (Gibbons et al. 1994), "capitalismo académico" (Slaughter y Leslie 1997) o "Triple Hélice" (Etzkowitz y Leydesdorf 1996). Para muchos, la ciencia deja de aportar únicamente reconocimiento y prestigio, y se abren las puertas a modos de remuneración basados en otras formas de capital (Braun 1998). En cualquier caso, estas transformaciones no afectan al componente vocacional de la actividad científica, al menos tal como lo hemos definido. La preferencia marcada por la actividad científica es compatible con un abanico amplio de motivaciones.

Como vemos, vocación y sistema de valores propio de la institución científica son dos aspectos de la actividad interrelacionados pero analíticamente diferenciables. El ordenamiento rígido de preferencias profesionales al que hemos llamado "vocación" implica la inmersión en la comunidad científica y la socialización en su propio ethos. Además, ese 
ethos conduce a menudo a un planteamiento de la profesión en términos vocacionales, o cuanto menos genera condiciones para la selección de aquellos que así se lo plantean. En cualquier caso, el ordenamiento rígido de preferencias profesionales es de hecho compatible con distintas configuraciones específicas del quehacer científico.

Responder a la pregunta de por qué esta institución selecciona positivamente a quienes experimentan la actividad en términos vocacionales excede las posibilidades de este artículo. Sin embargo, resulta plausible que, dado que la relación entre sociedad y científico (mediada por el Estado, las empresas, las agencias de investigación, etc.) puede ser considerada como una relación principal-agente (Fernández-Carro 2009), el predominio de las actitudes vocacionales sea, al menos en parte, el resultado de procesos de selección y regulación de la actividad que persiguen reducir el "riesgo moral del principal"2. La valoración formal de los méritos, y la valoración informal de las implicaciones prácticas de la vocación (entrega al trabajo, por ejemplo), pueden interpretarse como medios que persiguen evitar el problema de la "selección adversa"3.

\section{Las causas de la vocación}

A nuestro juicio, la idea generalizada de la vocación como tendencia inexplicable, prefijada e inmutable a determinado tipo de actividad, difumina y obscurece el verdadero papel del sujeto y la estructura social. El sujeto queda desdibujado, pues se le presenta como dominado por fuerzas internas presociales sobre las que no tiene control ni responsabilidad. La estructura y el contexto también quedan desdibujados, pues no se da cuenta de los condicionantes supraindividuales que canalizan y dan forma a la construcción de nuestras identidades.

Frente a esta idea de la vocación, entendemos que existen procesos estructuralmente condicionados en los que los sujetos dan forma a sus deseos. Así, la vocación sería el resultado final de un proceso en el que el sujeto ha encontrado un objeto-actividad que se demuestra por la experiencia como satisfactorio, formándose en ese encuentro el deseo de la realización de la actividad. El deseo de "hacer ciencia" se va formando gracias a experiencias satisfactorias "haciendo ciencia", y no las preexiste. Las y los jóvenes "llegan" o no a tener vocación científica en función de las experiencias por las que pasan en sus primeros contactos con el mundo de la producción de conocimiento. Dado que existe una desigualdad estructural entre mujeres y hombres, las actividades en las que unas y otros obtienen satisfacción y evidencia de su propia valía son distintas,

\footnotetext{
${ }^{2}$ Éste es el tipo de riesgo que asume un principal como consecuencia de que desconoce el nivel de esfuerzo real del agente. El agente tiene entonces la posibilidad de reducir su esfuerzo sin que ello afecte a su nivel de retribución.

${ }^{3}$ En la medida en que el principal no puede saber con certeza de qué tipo de agente se trata en cada caso (si se trata de un agente capaz, honrado, etc.), cabe la posibilidad de una "selección adversa", es decir, una selección que excluya a los mejores.
} 
de modo que se produce una diferencia de género en el tipo de "llamada" vocacional que "reciben" unas y otros.

\section{EL PERSONAL beCARIO EN LA UAB}

La carrera académica en la UAB, igual que en el resto de las universidades españolas, está regulada por la Ley orgánica 6/2001. En el marco anterior a esta ley, las trayectorias mediante las que se accedía a una plaza estable eran diversas. Las becas predoctorales podían ser seguidas de plazas de ayudante (con distintos niveles), y de éstas podía pasarse a plazas estables, como la de Titular de Universidad o de Escuela Universitaria. En aplicación de la ley actual, la UAB ha diseñado un modelo de carrera académica (aprobado por Consejo de Gobierno en diciembre de 20064) que comienza con la beca predoctoral (3-4 años), continua con una estancia postdoctoral en el extranjero (2 años), una reincorporación a la universidad mediante la plaza de lector (el equivalente a "ayudante doctor" en el resto de España, con una duración de 4-5 años), y el paso a una plaza de agregado (el equivalente a "contratado doctor" en el resto de España, con contrato indefinido).

Los datos nos señalan que la proporción de mujeres va menguando conforme avanzamos en estas categorías profesionales. Tal regularidad estadística parece un rasgo general de las universidades europeas (European Commission 2005), y la UAB no es una excepción, como podemos ver en el gráfico 1.

De todos los filtros que operan a lo largo de la trayectoria, en este estudio nos centraremos en aquellos que lo hacen en el proceso de disfrute de las becas.

El personal becario de la UAB se divide básicamente en dos colectivos: el personal becario predoctoral que disfruta de las becas FPI (Formación Profesional Investigador), FI (Formación de Investigadores), FPU (Formación del Profesorado Universitario), etc. PBP (Personal Becario Predoctoral) y el PAI (personal becario con becas de apoyo a la investigación, PAl en adelante). En la tabla 1 exponemos la distribución por sexo de estas becas.

Como vemos en la tabla 1, la presencia de hombres en las becas predoctorales es mayor a la esperada estadísticamente si consideramos la proporción de hombres titulados. Sea cual sea el mecanismo que actúa, existe un filtro en el proceso de selección a favor de los hombres, pues siendo un $32,7 \%$ de los titulados, obtienen el $50,8 \%$ de las becas predoctorales. Así pues, podríamos considerar las becas predoctorales como un terreno masculinizado. En cuanto al PAI, también podemos observar un proceso de masculinización, pero menor que el que tiene lugar en las becas predoctorales. Además, hemos de considerar que, mientras que de los hombres becados, el 65,9\% disfruta de una predoctoral y el $34,1 \%$ de una de apoyo a la investigación, de las mujeres becadas, el $59 \%$ disfruta de una predoctoral y el $41 \%$ de una de apoyo a la investigación.

\footnotetext{
${ }^{4}$ Puede consultarse en: https://intranet.uab.es/servlet/Satellite/el-nou-model-1194422294432.html
} 
Gráfico 1.

Proporción de mujeres y hombres a lo largo de la trayectoria académica en la UAB, según el sexo. Curso 2006-2007

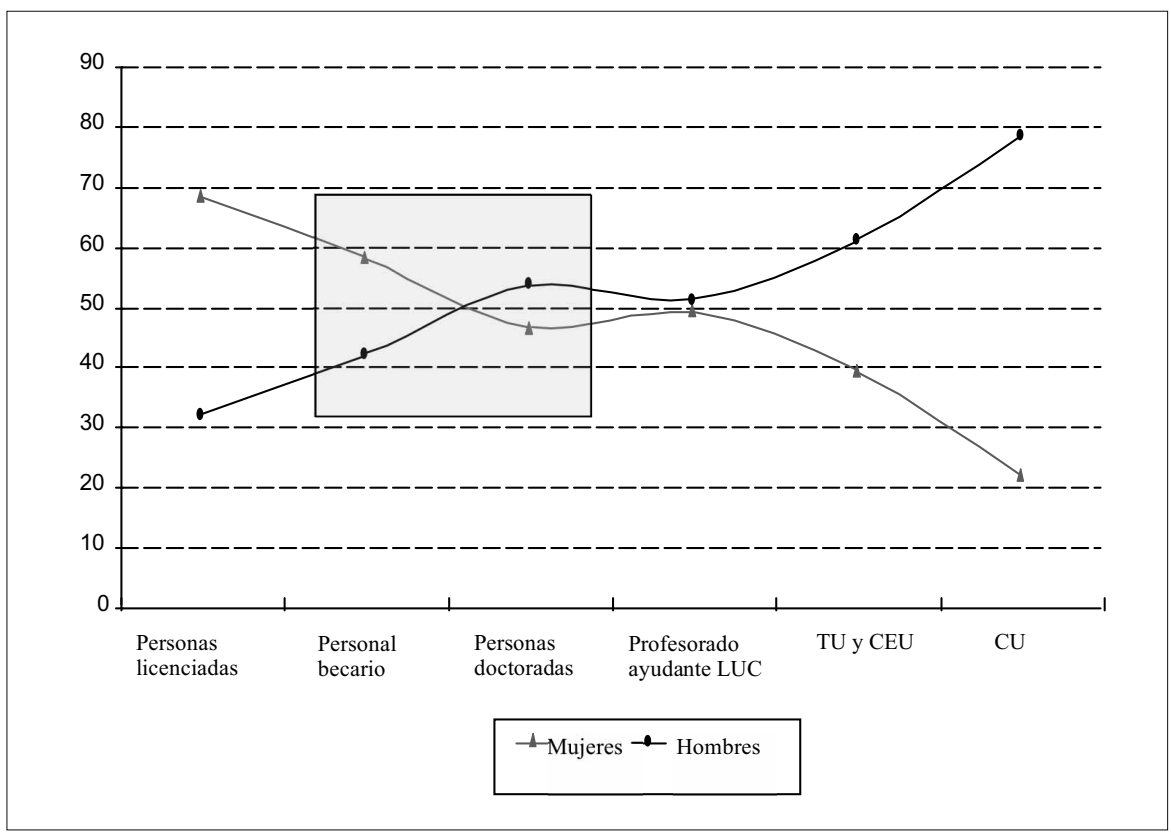

Nota: TU=titular de universidad; $\mathrm{CEU}=$ catedrático/a de escuela universitaria; $\mathrm{CU}=$ catedrático/a de universidad. Fuente: Curso 2006-2007 "UAB en xifres".

Tabla 1.

Becarios y titulados de la UAB según sexo (curso 2005-2006).

\begin{tabular}{l|c|c|c}
\hline & Mujeres & Hombres & Total \\
\hline \multirow{2}{*}{ Personal becario FPI } & 349 & 361 & 710 \\
\cline { 2 - 4 } & $49,2 \%$ & $50,8 \%$ & $100 \%$ \\
\hline \multirow{2}{*}{$\begin{array}{l}\text { Personal becario de apoyo a la } \\
\text { investigación }\end{array}$} & 243 & 187 & 430 \\
\hline \multirow{2}{*}{ Total personal becario } & $56,5 \%$ & $43,5 \%$ & $100 \%$ \\
\hline \multirow{2}{*}{ Total personas tituladas 04/05 } & 592 & 548 & 1140 \\
\cline { 2 - 4 } & $51,9 \%$ & $48,1 \%$ & $100 \%$ \\
\cline { 2 - 4 } & 3044 & 1479 & 4523 \\
\hline
\end{tabular}

Fuente: La UAB en xifres, 2005-2006. 


\section{Metodología}

Para este estudio de caso hemos desplegado una estrategia metodológica que combina técnicas cuantitativas y cualitativas. Para la cuestión específica que abordamos en este artículo, empelaremos el material obtenido mediante encuesta y entrevistas ${ }^{5}$.

\section{Entrevistas}

El objetivo principal de la realización de las entrevistas fue identificar aspectos clave de la trayectoria del personal becario, y sobre todo, la obtención de elementos que nos ayudaran en la comprensión de sus intereses, objetivos y preferencias. Para ello, se diseñaron entrevistas semiestructuradas alrededor de cuatro ejes temáticos: características personales y sociales de la persona entrevistada, proceso de decisión de optar por una beca, condiciones de trabajo y valoración del mismo, y expectativas de futuro y de continuidad en la carrera académica.

Se han realizado un total de 40 entrevistas. De ellas, 18 a personal becario de la UAB (10 con beca predoctoral, y 8 con beca de apoyo a la investigación) y 22 a profesorado estabilizado (de los cuales, 17 disfrutaron en el pasado de una beca predoctoral). Tanto para personal becario como para profesorado, la selección de entrevistados perseguía que se mantuviera un equilibrio entre hombres y mujeres, así como entre los distintos ámbitos de conocimiento (ciencias sociales, humanidades, ciencias experimentales, tecnología y ciencias de la salud).

Las encuestas fueron sometidas a un análisis de contenido realizado con Atlas-Ti. Esta herramienta sirvió principalmente para la clasificación y búsqueda temática de fragmentos de entrevista.

\section{Encuesta online}

Para la obtención de datos cuantitativos se optó por la realización de una encuesta online. Dado que la investigación se realizó en el marco del Observatorio para la Igualdad de la UAB y con apoyo institucional, se hacía posible la obtención del listado del personal becario censado en la UAB, así como sus direcciones de correo electrónico. De este modo, la encuesta online se convertía en la opción más indicada desde el punto de vista de la gestión de los recursos y el tiempo.

El análisis preliminar de los resultados obtenidos en las entrevistas aportó el material necesario para la elaboración de una tipología de respuestas, y ésta, a su vez, enriqueció el proceso de construcción de las preguntas cerradas que iban a formar parte de la encuesta.

\footnotetext{
${ }^{5}$ En el Anexo Estadístico pueden consultarse los perfiles del personal becario y docente entrevistado, así como los rasgos principales del personal becario que contestó la encuesta online.
} 
La encuesta tenía siete bloques: situación personal y familiar, situación profesional, acceso a la beca, actividades realizadas durante el disfrute de la beca, proceso de elaboración de la tesis doctoral, actividad docente, y valoración de la actividad como personal becario.

El cuestionario se envió a un total de 790 personas becarias, obteniendo 258 respuestas (190 con becas predoctorales, y 68 con becas de apoyo a la investigación), es decir, se obtuvo respuesta en un $32,7 \%$ de los envíos. El mensaje de correo que contenía el enlace a la encuesta se envió hasta tres veces a través del servicio centralizado de correo de la UAB. La encuesta se realizó entre mayo y junio de 2007.

Dadas las condiciones descritas, la realización de una encuesta online suponía inevitablemente la renuncia a la obtención de una muestra representativa del conjunto de la población de personal becario de la UAB. Este procedimiento implicaba inevitablemente la existencia de algunos sesgos de selección en la población encuestada. Por ámbitos de conocimiento, la diferencia proporcional entre el número de respuestas y el número de personas que conforman la plantilla es mínima, y por tanto no existe aquí un sesgo importante. El grupo de predoctorales sí está sobrerrepresentado en relación al grupo de apoyo a la investigación, del mismo modo que lo están las mujeres respecto de los hombres.

Por todo ello, el análisis de los datos cuantitativos no pretende en ningún caso ser extrapolable al conjunto de la población. Como veremos, las tablas presentadas apuntan a la existencia de relaciones entre variables que ya habían sido captadas por el análisis cualitativo. Pero, al no trabajar con una muestra, tan sólo podemos afirmar que tal relación se confirma para la población encuestada. En cualquier caso, a pesar de que no trabajamos con una muestra representativa, aportamos en las tablas los residuos estandarizados.

\section{ANÁLISIS DEL CASO}

\section{La vocación científica en el personal becario de la UAB}

Como hemos dicho, la vocación científica puede definirse como un orden rígido de preferencias profesionales y vitales. En este apartado presentamos de manera descriptiva las características de los órdenes de preferencias profesionales revelados en los discursos del personal becario sobre las expectativas y planes para el futuro ${ }^{6}$. En las entrevistas realizadas al PBP, las preguntas relacionadas con esta cuestión fueron las que generaron una diferencia mayor y más clara entre sexos.

Para los becarios, evocar el escenario posterior a la beca supone evocar la beca postdoctoral y la estancia en el extranjero que conlleva y que, a su vez, parece prerre-

\footnotetext{
${ }^{6}$ Nos centramos en la vocación como un orden rígido de preferencias, pero por una cuestión de espacio, no entraremos al estudio de su dimensión práctica e identitaria.
} 
quisito para la estabilización en la universidad. Todos ellos afirman que les gustaría dedicarse a la docencia, y que ésta se realizase en la universidad. Es, por tanto, un objetivo deseado alrededor del cual diseñan sus planes futuros.

Los resultados de la encuesta online apuntan en la misma dirección, es decir, nos permiten decir que tal diferencia existe entre la población encuestada:

Tabla 2.

Creencia de que se seguirá en la universidad una vez acabada la beca, según sexo (PBP de la UAB)

\begin{tabular}{llrrr}
\hline & & Mujeres & Hombres & Total \\
\hline \multirow{2}{*}{$\begin{array}{l}\text { Sí, seguiré en la } \\
\text { universidad }\end{array}$} & $\mathrm{n}$ & 40,0 & 33,0 & 73,0 \\
\cline { 2 - 5 } & $\%$ col & 35,1 & 43,4 & 38,4 \\
\cline { 2 - 5 } & Res.Est. & $-0,6$ & 0,7 & \\
\hline \multirow{2}{*}{$\begin{array}{l}\text { No seguiré en la } \\
\text { universidad }\end{array}$} & $\mathrm{n}$ & 70,0 & 41,0 & 111,0 \\
\cline { 2 - 5 } & $\%$ col & 61,4 & 53,9 & 58,4 \\
\hline \multirow{3}{*}{ NS/NC } & Res.Est. & 0,4 & $-0,5$ & \\
\cline { 2 - 5 } & $\mathrm{n}$ & 4,0 & 2,0 & 6,0 \\
\hline \multirow{2}{*}{ Total } & Res.Est. & 0,2 & $-0,3$ & 3,2 \\
\hline
\end{tabular}

Fuente: Encuesta online.

Esto no quiere decir que no se consideren las dificultades que existen para la consecución del objetivo, pues se muestran perfectamente conscientes de ellas. Pero, aunque se señale lo elevado del coste, se afirma al tiempo la voluntad de asumirlo y de perseguir el fin que se han planteado. Su vínculo con la actividad es asertivo: a pesar de su conciencia de las dificultades, tratan de hacer que aquello que quieren que les pase, les pase efectivamente.

Ninguna becaria entrevistada, sin embargo, expresa su deseo de hacer una estancia postdoctoral en el extranjero. Las entrevistadas que sí aluden a esta posibilidad, la plantean como una apuesta insegura que aún no ven con suficiente claridad si habrían de realizar, o que ya ven que descartarán. Sus planes se centran fuera de la universidad, y se evidencia un menor entusiasmo en la continuación de la carrera académica. Lo destacado prioritariamente es la dificultad de obtener una plaza, la falta de posibilidades, la dureza de los obstáculos, etc. Cuando reflexionan sobre lo que efectivamente les espera después de la beca, su narración circula ya por escenarios ajenos a la universidad: hablan de las dificultades para encontrar trabajo, de las dudas respecto de la utilidad del doctorado para ello, de la posibilidad de pasar a una etapa peor, etc. 
En este contexto, parece que lo masculino es no renunciar ni rendirse, y aunque las dificultades se vean claras, insistir en la persecución del objetivo, mientras que lo femenino consiste en redefinir los objetivos en un proceso siempre abierto de reformulación contextualizada de las preferencias. Esta diferencia es propia de una situación de desigualdad. Resistirse y pelear sólo tiene sentido para aquellas personas que se encuentran en una posición estructural en la que, o bien se perciba alguna posibilidad de éxito, o bien se haga posible obtener algún placer en su búsqueda.

En definitiva, la voluntad de permanecer en la academia es menos generalizada entre las mujeres, y aunque resulta evidente que existen otros factores influyendo en la determinación del contingente de mujeres que abandonan la academia tras la beca, creemos que este tiene una importancia destacable. Por ello se hace necesario estudiar los procesos causales que han dado como resultado esta diferencia en la voluntad de permanencia y el entusiasmo con la carrera científica.

\section{Mecanismos situacionales en la construcción de la vocación científica en el per- sonal becario de la UAB}

A continuación presentamos los distintos mecanismos situaciones que hemos identificado a través del análisis de las encuestas y las entrevistas.

\section{La privación relativa}

El primero de los mecanismos que parece estar contribuyendo a la diferencial construcción de los órdenes de preferencias de mujeres y hombres, es el mecanismo de la "privación relativa" (Merton 1948). Según Merton, la formación de creencias relativas a las propias oportunidades de éxito en una organización, así como los niveles de satisfacción personal en la misma, son en gran medida dependientes del grupo que se toma como referencia a la hora de evaluar la propia situación. En el caso de la UAB, el PAI, que es un colectivo menos masculinizado que el PBP, parece tomar a este último como referencia. Esto puede ayudarnos a explicar la aparente paradoja que se produce entre la conciencia de la fortuna de disfrutar de esta beca (todas las personas entrevistadas son conscientes de que es un mejor escenario para doctorarse que la empresa privada) y la valoración tan crítica que realizan del hecho de ser personal becario. La existencia de un "otros" muy similar al "nosotros" pero con condiciones de trabajo y perspectivas mucho más favorables, está en la base de esta actitud crítica. Tomar como referencia al PBP se traduce, para el PAI, en una creencia firme en las escasas posibilidades de éxito en la academia, y en una actitud más crítica y distante respecto a la universidad. Al fin y al cabo, las personas que disfrutan de una beca predoctoral se encuentran en una posición en la que su vínculo con la docencia y la investigación puede realizarse de modo más provechoso: el PBP suele estar más libre de labores de gestión y administración, tiene la posibilidad de enfrentarse a la docencia, normalmente investiga en un proyecto en el marco del cual realiza su tesis, etc. En un contexto de generalizada dificultad, el PAI 
tiene menos razones para el optimismo que el PBP. Así, la formación de creencias sobre las propias oportunidades en el futuro toma formas más pesimistas entre el PAl, que es el colectivo becario en el que la presencia de mujeres es mayor. En general, estas becas no son un terreno fértil para el nacimiento de la vocación científica.

En el caso del PBP, el grupo que toman como referencia para la evaluación de su situación es el del personal docente. Pero, en este caso, resulta bastante plausible que los hombres tomen como referencia a los profesores, y al menos para algunas cuestiones fundamentales, las becarias tomen como referencia a las profesoras. Esto tiene implicaciones prácticas muy importantes. En el caso de los becarios, lo que observan es un futuro posible al que quizá se puede llegar con esfuerzo.

Becario PR 27: "Hago cálculos: me gustaría ser profesor de la UAB. A mi director le han dado la plaza fija y tiene 35 años, y yo tengo 25 . Me quedan 10 años de seguir aquí...".

Como vemos en este fragmento, la referencia de la estabilización de su director es interpretada en términos cronológicos, como si fuese una cuestión de tiempo. Así, tiene sentido que la tenacidad se convierta en la actitud predominante en los becarios.

En el caso de las becarias, la referencia de las mujeres en sus departamentos puede ejercer una influencia disuasoria en la formación de ilusiones respecto del futuro. Para empezar, la desaparición de las mujeres en las categorías superiores puede actuar como una señal que indique a las becarias que sus posibilidades de éxito son especialmente limitadas. Por otra parte, las especiales dificultades de las mujeres que sí han conseguido estabilizarse pueden servir también como estímulo a la renuncia. Esto es especialmente cierto respecto de la maternidad, tal como veremos en el próximo apartado.

Es posible, en definitiva, que el grupo que se toma como referencia para la valoración condicione las creencias respecto de las propias oportunidades. Más adelante veremos cómo estas creencias pueden condicionar los órdenes de preferencias para el futuro.

\section{Los costes de la incertidumbre}

En las actuales condiciones, las mujeres pueden experimentar como más costoso 0 menos asumible el indefinidamente prologando periodo de incertidumbre que precede a una posible estabilización en la universidad. De hecho, las dudas asociadas a la incertidumbre están ya presentes en el momento de solicitud de la beca. Esto se confirma para la población encuestada, como podemos ver en la siguiente tabla:

\footnotetext{
${ }^{7}$ Los códigos que emplearemos en las citas de las entrevistas son los siguientes: PR: personal becario predoctoral; Al: personal becario de apoyo a la investigación. En el anexo pueden consultarse los perfiles del personal entrevistado.
} 
Tabla 3.

Tipos de dudas para pedir una beca, según sexo. PBP.

\begin{tabular}{llrrr}
\hline Tipos de dudas & & Mujeres & Hombres & Total \\
\hline \multirow{2}{*}{$\begin{array}{l}\text { Dudaba si tenía sentido dedicar tanto } \\
\text { esfuerzo sin nada asegurado para el futuro }\end{array}$} & $\mathrm{n}$ & 24,0 & 8,0 & 32,0 \\
\cline { 2 - 5 } & $\%$ col & 21,1 & 10,5 & 16,8 \\
\cline { 2 - 5 } & Res.Est. & 1,1 & $-1,3$ & \\
\hline \multirow{2}{*}{$\begin{array}{l}\text { Dudaba si no era más rentable pasar a la } \\
\text { empresa privada }\end{array}$} & $\mathrm{n}$ & 4,0 & 7,0 & 11,0 \\
\cline { 2 - 5 } & $\%$ col & 3,5 & 9,2 & 5,8 \\
\cline { 2 - 5 } & Res.Est. & $-1,0$ & 1,2 & \\
\hline \multirow{2}{*}{$\begin{array}{l}\text { Dudaba si quería desvincularme durante } \\
\text { unos cuantos años del mundo laboral }\end{array}$} & $\mathrm{n}$ & 8,0 & 6,0 & 14,0 \\
\cline { 2 - 5 } & $\%$ col & 7,0 & 7,9 & 7,4 \\
\cline { 2 - 5 } & Res.Est. & $-0,1$ & 0,2 & \\
\hline \multirow{2}{*}{$\begin{array}{l}\text { Dudaba si la dedicación a las tareas de } \\
\text { becario me permitiría o dificultaría hacer }\end{array}$} & $\mathrm{n}$ & 0,0 & 2,0 & 2,0 \\
\cline { 2 - 5 } la tesis y/o tesina & $\%$ col & 0,0 & 2,6 & 1,1 \\
\cline { 2 - 5 } & Res.Est. & $-1,1$ & 1,3 & \\
\hline \multirow{3}{*}{ NS/NC } & $\mathrm{n}$ & 78,0 & 53,0 & 131,0 \\
\cline { 2 - 5 } & $\%$ col & 68,4 & 69,7 & 68,9 \\
\cline { 2 - 5 } & Res.Est. & 0,0 & 0,1 & \\
\hline \multirow{2}{*}{ Total } & $\mathrm{n}$ & 114,0 & 76,0 & 190,0 \\
\cline { 2 - 5 } & $\%$ fila & 60,0 & 100,0 \\
\hline
\end{tabular}

Fuente: Encuesta online.

El periodo más indicado para la reproducción coincide con la etapa de inestabilidad laboral que puede suceder al disfrute de la beca, lo que exigiría o bien una renuncia, 0 bien una arriesgada postergación de la maternidad. Además, las condiciones en las que ésta se ejerce actualmente no favorecen su adecuada conciliación con la vida laboral. En el caso que analizamos, la maternidad sólo está presente como un plan futuro. Ese plan, sin embargo, condiciona el modo en que se toman decisiones y se experimenta el presente, y sobre todo, el modo en que se diseñan las apuestas y pretensiones para el futuro. La referencia de las mujeres estabilizadas vuelve aquí a ser relevante. Así se expresaba una becaria:

Becaria Al 2: "Esto [la carrera académica] es muy absorbente, todo gira alrededor de esto. Por ejemplo, en la maternidad. Las pocas profesoras que hay en [...], las que han sido madre lo han sido a partir de los 40 , y dicen que lo hicieron para poner todo el esfuerzo en la carrera. $Y$ algunas renunciaron a la maternidad. Tienes que sacrificar muchas cosas. Y no es una apuesta segura". 
La apuesta por la estabilización puede suponer asumir una ruptura con la vida cotidiana para marcharse al extranjero con una beca postdoctoral. Además, la apuesta es arriesgada, pues no está asegurada la incorporación a la vida académica a la vuelta. La posibilidad de tener que abrirse paso si las cosas no salen según lo planeado configura un escenario futuro de considerable dificultad.

Es más que posible que la menor implicación masculina en los planes de paternidad contribuya a que los becarios evalúen como menos costoso asumir un nuevo periodo de incertidumbre y una postergación de la estabilización en un puesto o territorio. Por su parte, las mujeres parecen estar fijándose algunos topes más allá de los cuales la inversión de tiempo y esfuerzo en la academia pasaría a considerarse menos atractiva para el ejercicio de la maternidad que la inversión realizable en la empresa privada o en la Administración pública. En el contexto actual, los planes de maternidad condicionan el grado del riesgo asumible en un periodo crítico del ciclo vital. Esta cuestión quedó bien patente en las entrevistas a profesoras. Por ejemplo:

Profesora 4: "Es una carrera de fondo, que quien la aguanta es mayoritariamente un hombre, y es muy larga, y a una mujer le suena el reloj biológico y se le plantea la maternidad, y en una situación no estable es muy difícil, y hay muchas chicas que acaban en un instituto [como profesoras], y quienes aguantan son ellos. Ellos que aún no tienen una plaza estable, pero que pueden esperar a la cuarentena para la plaza".

La cuestión de la maternidad puede estar contribuyendo a acrecentar el coste que supone la incertidumbre propia de la etapa previa a la estabilización en la academia, pero también puede condicionar las creencias acerca de las propias posibilidades de éxito una vez estabilizada la plaza. Aquí, de nuevo, la referencia de las profesoras puede ser fundamental para las becarias. Tres de las profesoras entrevistadas, por ejemplo, hicieron referencia a la dificultad de compaginar la vida familiar y la profesional. En un caso, se afirma expresamente que el cuidado de hijas e hijos supone una merma en la actividad investigadora, y en los otros, se alude a la dificultad de preparar una oposición o una defensa de tesis cuando se tienen hijas e hijos que cuidar o se está embarazada.

En definitiva, los costes de asumir el prolongado período de incertidumbre antes de la estabilización son mayores para las mujeres, en la medida en que interfieren con los planes de una maternidad que, además, en sí misma puede suponer en las actuales condiciones un freno o una dificultad extra para la consecución del éxito (medido, por ejemplo, a través de las evaluaciones de la calidad del currículum que realizan las agencias de evaluación).

\section{Experiencias y estereotipos}

Obviamente, las creencias sobre la propia valía para el trabajo académico condicionan en gran medida la probabilidad de la formación del orden rígido de preferencias al que hemos llamado "vocación". A su vez, esas creencias son muy dependientes de dos fac- 
tores: las experiencias por las que se pasa durante el disfrute de la beca, y los estereotipos de género vigentes sobre la actividad científica.

Si damos por buena la idea de que la vocación no es un atributo presocial, sino el resultado de una biografía determinada, parece lógico pensar que los condicionantes estructurales que facilitan experiencias satisfactorias y enriquecedoras en un ámbito sean las que estimulen el nacimiento del deseo de su realización. Por ello, condiciones diferentes para mujeres y hombres pueden estar determinando la mayor o menor presencia de esta vocación en cada uno de los colectivos.

En este punto, la diferencia entre el PBP y el PAI vuelve a ser fundamental. El colectivo menos masculinizado de los dos es precisamente aquél que se caracteriza por tareas diarias más alejadas de la investigación. Entre el PAI resultan frecuentes las labores administrativas, y la participación en proyectos de temática ajena a su tesis doctoral, lo que supone en general una necesidad de arrinconar el proceso de elaboración de la tesis a los momentos que la beca deja libres. Evidentemente, la posibilidad de disfrutar de la investigación queda considerablemente distorsionada. Además, este colectivo se caracteriza por no ejercer labores docentes, de modo que también tiene vetada la posibilidad de experimentar el placer o la frustración de la docencia. Todos estos condicionantes generan menor posibilidad de identificar la actividad académica como una actividad en la que uno o una se siente útil y realizado.

Dentro del colectivo de PBP, no existen diferencias en la carga de trabajo que asumen mujeres y hombres (aunque las profesoras estabilizadas sí parecen haber asumido una carga extra cuando disfrutaron de sus becas), con la importante excepción de la actividad docente. Hay que señalar que todos los becarios entrevistados, excepto uno, han realizado el máximo de docencia establecido. Entre las becarias predoctorales, sin embargo, la mayoría de las entrevistadas no desarrollaron ningún tipo de actividad docente durante el curso. Además, las que realizaron docencia tan sólo asumieron la mitad de la carga que les correspondía. Es decir, que en general, la dedicación docente es más generalizada y más intensa en los becarios que en las becarias ${ }^{8}$. Entre la población encuestada encontramos la misma tendencia, ver tabla 4.

Esto puede dificultar las posibilidades de éxito de la carrera académica de las becarias. En primer lugar, porque una vez acabada la beca es posible que se encuentren en peores condiciones para competir por un puesto con hombres que sí tienen experiencia docente. En un período de cuatro años, la ventaja en investigación que pueden haber desarrollado ellas al no haber tenido que dedicar esfuerzos a la docencia, puede no ser tan determinante como para asegurarles una ventaja, y sin embargo la carencia de experiencia docente sí les puede situar en peor posición. En segundo lugar, la distan-

\footnotetext{
${ }^{8}$ Esta diferencia entre becarias y becarios no se explica por la pertenencia a ámbitos de conocimiento distintos. Las becarias entrevistadas que no han tenido dedicación docente en el curso presente son Tecnológicas, Humanidades y Ciencias de la Salud, mientras que el único hombre que no hizo docencia es de Ciencias Sociales.
} 
Tabla 4.

Dedicación a actividades docentes, según sexo. PBP de la UAB.

\begin{tabular}{llccc}
\hline & & Mujeres & Hombres & Total \\
\hline \multirow{3}{*}{ No hago actualmente } & $\mathrm{n}$ & 57 & 34 & 91 \\
\cline { 2 - 5 } & $\%$ col & 50,0 & 44,7 & 47,9 \\
\cline { 2 - 5 } & Res.Est. & 0,3 & $-0,4$ & \\
\hline \multirow{3}{*}{ Sí que hago, en el marco de la beca } & $\mathrm{n}$ & 54 & 42 & 96 \\
\cline { 2 - 5 } & $\%$ col & 47,4 & 55,3 & 50,5 \\
\cline { 2 - 5 } & Res.Est. & $-0,5$ & 0,6 & \\
\hline \multirow{3}{*}{ NS/NC } & $\mathrm{n}$ & 3 & 0 & 3 \\
\cline { 2 - 5 } & $\%$ col & 2,7 & 0,0 & 1,6 \\
\hline \multirow{2}{*}{ Total } & Res.Est. & 0,9 & $-1,1$ & 190 \\
\hline
\end{tabular}

Fuente: Encuesta online

cia respecto de la actividad docente puede tener también un impacto en el proceso de definición del orden de preferencias de las mujeres, en la medida en que, como venimos diciendo, las preferencias se definen a menudo en el propio desarrollo de las actividades.

Los estereotipos pueden marcar también los procesos de autoevaluación de las capacidades. Históricamente, la actividad científica ha sido considerada masculina, y la comunidad científica ha sido muy hostil a la entrada de mujeres. En la actualidad, el sesgo masculino en los contenidos y procedimientos de la actividad científica, así como en los procesos de evaluación (Izquierdo et al. 2008), pueden tener impacto en la formación de creencias de las propias mujeres respecto de su adecuación y valía para las labores académicas. Una regularidad bien establecida en nuestros datos es que los hombres presentan sus méritos como un "dato", como una realidad objetivada en su currículum, sus publicaciones, etc., mientras que, entre las mujeres, es más frecuente presentar el propio mérito como algo evidenciado a través del reconocimiento que de él hicieron otras personas. Por ejemplo, así respondían algunos becarios a la pregunta de por qué creían que habían ganado su beca:

Becario PR 2: "Porque me la merecía, por nota, etc. [...] si te la dan es porque tenías mejor nota y es justo".

Becario PR 3: "... me la dieron [la beca] de la UAB por expediente".

Becario PR 5: "Porque había un proyecto global ya formalizado y yo estaba enmarcado dentro, y después había un factor currículum. [...] con un 3 [en el expediente] es muy probable que te la den". 
Algunas becarias respondían en los mismos términos, pero otras apuntaban no tanto a su mérito, sino a la posición o la valoración del mérito realizada por personas cercanas:

Becaria PR 1: "... el que escogía era mi jefe. [...]. Yo ya hacía tres años que estaba aquí, y él quería que me la concediesen a mí".

Becaria PR 5: "Porque mi directora que era investigadora principal del proyecto seleccionaba y ella pensaba que yo era la mejor candidata".

Becaria Al 1: "Porque ya trabajaba con ellos, y porque han creído en mi, ya habían visto mi dinámica de trabajo".

En estos fragmentos, podemos ver que los becarios no reconocen ni dan relevancia al hecho de que los apoyos son necesarios en la obtención de la beca. Podría pensarse que, hasta cierto punto, dan por supuestos los privilegios de los que disfrutan. Tal desconsideración, paradójicamente, puede aportar una dosis extra de motivación.

En el caso de las mujeres, la apertura y sensibilidad a los juicios ajenos puede contribuir a la construcción de procesos de autoevaluación dependientes en exceso del contexto. Es decir, las becarias sí reconocen la importancia de los apoyos, pero el resultado de tal consideración puede tener, en un contexto de desigualdad, un impacto negativo en la motivación.

Si el juicio acerca del propio valor es dependiente del contexto, y si el contexto es predominantemente masculino, resulta más probable que las autoevaluaciones reflejen en cierta medida los sesgos y estereotipos culturales del entorno, tal como ha mostrado experimentalmente Correll (2004). De este modo, el estereotipo de la ciencia como actividad masculina puede traducirse en una autoevaluación más severa en las mujeres, y más generosa en los hombres.

Esta idea puede verse confirmada de modo indirecto observando la llustración 2 y la Tabla 5. Como vemos, unos expedientes inferiores en promedio no impiden que los becarios predoctorales encuestados señalen los méritos propios como causa de la obtención de su beca más frecuentemente que las becarias.

Esto, a su vez, genera diferencias en el grado de satisfacción que puede obtenerse de la actividad docente e investigadora, pues el placer es mayor cuanta más seguridad tiene una persona en su capacidad de hacerlo bien. Además, la conciencia de que lo obtenido no depende de los propios actos sino de la voluntad de los demás produce un menor placer que la creencia de que todo lo obtenido es el producto del propio mérito. De nuevo, el reconocimiento de la necesidad de apoyos diluye el potencial placentero de la actividad.

Parece claro, por tanto, que estos procesos de autoevaluación culturalmente condicionados contribuyen a la diferencial construcción del orden de preferencias profesionales de becarias y becarios. 
Gráfico 2.

Expediente académico según sexo. PBP.

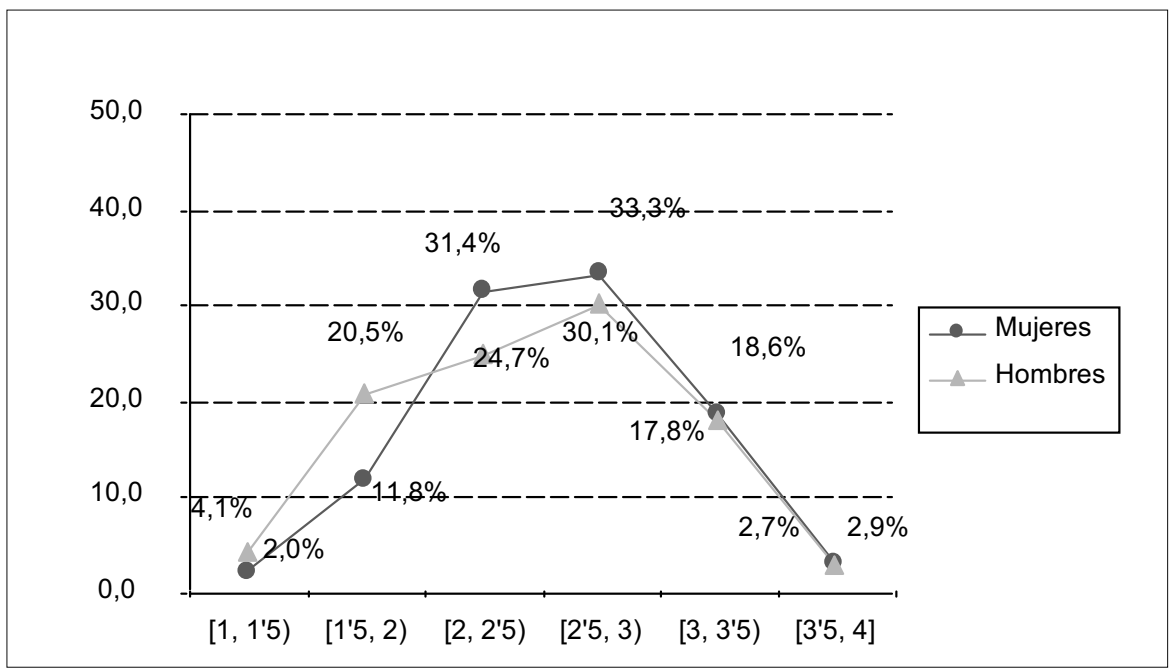

Nota: Hay 15 casos (7'8\%) que han respondido NS/NC.

Fuente: Encuesta online.

Tabla 5.

Motivos subjetivos de obtención de la beca según sexo (PBP)

\begin{tabular}{llccc}
\hline \multirow{2}{*}{ Motivos } & & Mujeres & Hombres & Total \\
\hline \multirow{3}{*}{ Por méritos propios } & $\mathrm{n}$ & 61 & 47 & 108 \\
\cline { 2 - 5 } & $\%$ col & 53,5 & 61,8 & 56,8 \\
\cline { 2 - 5 } & Res.Est. & $-0,5$ & 0,6 & \\
\hline \multirow{3}{*}{ Por méritos o necesidades del grupo } & $\mathrm{n}$ & 42 & 21 & 63 \\
\cline { 2 - 5 } & $\%$ col & 36,8 & 27,6 & 33,2 \\
\cline { 2 - 5 } & Res.Est. & 0,7 & $-0,8$ & \\
\hline \multirow{3}{*}{ Por afinidad entre las partes } & $\mathrm{n}$ & 6 & 7 & 13 \\
\cline { 2 - 5 } & $\%$ col & 5,3 & 9,2 & 6,8 \\
\cline { 2 - 5 } & Res.Est. & $-0,6$ & 0,8 & 6 \\
\hline \multirow{2}{*}{ NS/NC } & $\mathrm{n}$ & 5 & 1 & 3,2 \\
\cline { 2 - 5 } & $\%$ col & 4,4 & 1,3 & 190 \\
\cline { 2 - 5 } Total & Res.Est. & 0,7 & $-0,9$ & 100 \\
\hline
\end{tabular}

Fuente: Encuesta online.

RIS, VOL. 68. No 2, MAYO-AGOSTO, 399-428, 2010. ISSN: 0034-9712. DOI: 10.3989/ris.2008.06.19 


\section{Preferencias adaptativas y profecía autocumplida}

La diferencia en los grupos de referencia según sexo y tipo de beca que se disfruta, la diferencia en el grado y el coste de la incertidumbre para mujeres y hombres, y las diferentes experiencias y estereotipos que condicionan la propia autoevaluación, contribuyen a la formación del orden de preferencias profesionales de becarias y becarios.

En el caso de las mujeres, todos estos factores dan forma a la sospecha de un más que previsible fracaso. Para rebajar la intensidad emocional del mismo, entra en juego el mecanismo de las "preferencias adaptativas" (Elster 1988): se pretende que no se desea aquello que se cree que no se puede obtener, y tal pretensión se apoya en una degradación del valor que atribuimos a aquello que no podemos obtener. Como ya hemos dicho, en sus discursos sobre lo que desean para el futuro, las becarias parecen estar lidiando con un deseo que tratan de reorientar (por ejemplo, de la docencia universitaria a la secundaria, o de la investigación en la universidad a la investigación en empresas 0 institutos) o que afirman no tener (y en ese caso la docencia no interesaría tanto como para realizar una apuesta de resultado tan incierto). Podemos verlo ejemplificado en los siguientes fragmentos de entrevista:

Becaria PR 2: "Me gustaría encontrar trabajo en el sector privado. Aquí lo veo difícil porque no salen plazas, al menos por aquí. Hacer un postdoc no me apetece mucho". Becaria PR 4: "... el tema de las posdoc no lo veo claro porque supone apostar ya por una carrera académica que no veo muy clara, al menos en la UAB [...]. Es una carrera de obstáculos agónica y no sé si estoy dispuesta a hacerlo".

Becaria PR 5: "Ahora trabajar en una empresa es lo que me apetece más. Aquí en el departamento como profesor es complicadísimo quedarse, tendría que hacer un postdoc fuera, y acreditarme, y una serie de pasos, y la docencia tampoco es que me motive tanto".

Becaria Al 5: "O continuar en la pública como profesora de secundaria, o empresa privada y trabajar en [...] algo relacionado con el doctorado".

En el caso de los hombres, la situación en la que se encuentran estimula la construcción de una subjetividad caracterizada por el fortalecimiento de la voluntad de permanencia en la universidad. Aunque las posibilidades de estabilización en la academia son escasas para todos, los becarios tienen referencias y modelos cercanos con los que identificarse, tienen una mayor posibilidad y predisposición a asumir el coste de la incertidumbre y las posibles rupturas de la vida cotidiana que se producen por las estancias postdoctorales en el extranjero, se enfrentan a lo que entienden que es un grado menor de incertidumbre, y se encuentran en una posición que favorece la acumulación de experiencias potencialmente satisfactorias en la investigación y la docencia. Al contrario que en las mujeres, el conjunto de oportunidades de los hombres estimula la construcción de un orden de preferencias profesionales en el que la opción de invertir esfuerzo en el objetivo de la permanencia en la academia se convierte en la preferida, 
a mucha distancia de otras opciones como la empresa privada, las oposiciones a la administración pública, etc. Así puede comprobarse en la claridad con la que trazan sus planes futuros:

Becario PR 1: "La idea sería continuar investigando. Acabar la tesis e irme de postdoc...".

Becario PR 2: "Me gustaría hacer una postdoc [...] Me gustaría muchísimo ser profesor. Si pudiese quedarme como profesor de mi licenciatura y aquí en la UAB, sería la mejor opción de todas".

Becario PR 5: "Pedir una beca postdoc e irme fuera. Quiero hacer que la tesis... de los distintos capítulos sacar muchos artículos y publicar mucho".

Si el conjunto de oportunidades de las mujeres activa el mecanismo de las preferencias adaptativas, y las mujeres construyen un discurso en el que pretenden que no desean la permanencia en la academia, éste mecanismo situacional genera un conjunto de acciones por parte de las mujeres que activa otro mecanismo de tipo "transformacional" (Hedstrom y Swedberg 1996; 1998), es decir, que explica la transición micro-macro. Se trata del mecanismo de la "profecía autocumplida" (Merton 1948). En este caso, el contexto favorece que las mujeres elaboren la creencia de que sus posibilidades de éxito en la academia son escasas, o más escasas que las de los hombres. Esta creencia, tenga o no valor como descripción objetiva de la situación, contribuye a su propia verificación, pues incentiva en las mujeres la apuesta por opciones profesionales ajenas al entorno universitario.

\section{CONCLUSIONES}

La ausencia de las mujeres en los escalones más altos de las plantillas del personal académico es un fenómeno ampliamente constatado, pero los marcos explicativos de esta regularidad estadística todavía no han arrojado suficiente luz sobre los mecanismos causales que la generan. En este artículo hemos pretendido contribuir a la identificación de algunos de los factores causales mediante el análisis de un caso. En concreto, nos hemos centrado en un punto especialmente importante de la trayectoria académica: el disfrute de las becas predoctorales o de apoyo a la investigación. Basándonos en el análisis de entrevistas realizadas a personal becario y profesorado de la $U A B$, y en los datos de una encuesta online al personal becario de la misma universidad, hemos tratado de poner de relieve la importancia de los procesos diferenciales de construcción de los órdenes de preferencias profesionales de mujeres y hombres.

La vocación científica, que aquí ha sido definida como un orden rígido de preferencias profesionales, no es un atributo presocial, sino el resultado de creencias y experiencias condicionadas en su contenido por la posición estructural que se ocupa 
en unas relaciones de desigualdad. Las condiciones y dedicaciones laborales, los estereotipos vigentes, los modelos de referencia que se adoptan y los costes percibidos de las distintas opciones de futuro son algunas de las variables que puede ayudarnos a comprender por qué la experiencia de la beca conduce a una configuración de la subjetividad distinta en mujeres y hombres. En definitiva, hemos podido comprobar que en el caso analizado las posiciones de becarias y becarios estimulan una desigual construcción de sus órdenes de preferencias profesionales, alimentando la vocación científica más frecuentemente entre los becarios que entre las becarias. Aunque en la academia la vocación científica no sea un factor de éxito suficiente, sin duda contribuye en gran medida a la probabilidad de progresar profesionalmente, y de ese modo, puede ayudarnos a explicar en algún grado la progresiva desaparición de las mujeres de la actividad científica que se produce cuanto más alta es la categoría del profesorado.

\section{Anexo estadístico}

Tabla 6.

Rasgos más destacados del personal becario encuestado (2007)

\begin{tabular}{|c|c|c|}
\hline & Mujeres & Hombres \\
\hline Sexo & Mujer $(62,8 \%)$ & Hombre $(37,2 \%)$ \\
\hline Orientación de la beca* & $\begin{array}{c}\text { Predoctoral } \\
\text { (70,4\% de les mujeres becarias) }\end{array}$ & $\begin{array}{c}\text { Predoctoral } \\
\text { (79,2\% de los hombres becarios) }\end{array}$ \\
\hline Edad media & 27,4 años & 27,6 años \\
\hline Ámbito de conocimiento & $\begin{array}{l}\text { Ciencias Experimentales i } \\
\text { Tecnologías }(50,3 \%)\end{array}$ & $\begin{array}{l}\text { Ciencias Experimentales } \\
\text { y Tecnologías }(45,3 \%)\end{array}$ \\
\hline Sueldo medio" & $1.019,8 € /$ mes & $987,0 € /$ mes \\
\hline Tipo de hogar & Vive en pareja $(47,5 \%)$ & $\begin{array}{l}\text { Vive solo o con compañeros } \\
\qquad(39,6 \%)\end{array}$ \\
\hline Fuente principal de ingresos & Una misma $(31,5 \%)$ & Uno mismo $(43,8 \%)$ \\
\hline
\end{tabular}

* Señala el objetivo de la beca: realizar el doctorado o dar apoyo a la investigación.

"*ara realizar la media salarial se han tenido en cuenta los casos con sueldo igual o superior a $800 € /$ mes, de acuerdo a las características de dedicación a tiempo completo.

Fuente: Elaboración propia a partir del cuestionario online, 2007. 
Tabla 7.

Perfil del personal becario predoctoral entrevistado

\begin{tabular}{lccc}
\hline & Ámbito de conocimiento & Edad & Año de obtención beca \\
\hline Becaria 1 & Tecnológicas & 32 & 2005 \\
\hline Becaria 2 & Experimentales & 29 & 2004 \\
\hline Becaria 3 & Humanidades & 26 & 2005 \\
\hline Becaria 4 & Sociales & 28 & 2004 \\
\hline Becaria 5 & Salud & 26 & 2004 \\
\hline Becario 1 & Experimentales & 27 & 2003 \\
\hline Becario 2 & Salud & 25 & 2004 \\
\hline Becario 3 & Tecnológicas & 26 & 2005 \\
\hline Becario 4 & Sociales & 26 & 2005 \\
\hline Becario 5 & Humanidades & 26 & 2004 \\
\hline
\end{tabular}

Tabla 8.

Perfil del personal becario de apoyo a la investigación entrevistado

\begin{tabular}{lccc}
\hline & Ámbito de conocimiento & Edad & Año de obtención beca \\
\hline Becaria 1 & Experimentales & 29 & 2006 \\
Becaria 2 & Humanidades & 24 & 2006 \\
Becaria 3 & Salud & 24 & 2005 \\
Becaria 4 & Sociales & 25 & 2007 \\
Becaria 5 & Tecnológicas & 31 & 2005 \\
Becario 1 & Salud & 28 & 2007 \\
Becario 2 & Sociales & 26 & 2007 \\
Becario 3 & Tecnológicas & 24 & 2007 \\
\hline
\end{tabular}


Tabla 9.

Perfil del profesorado entrevistado

\begin{tabular}{|c|c|c|c|c|}
\hline & $\begin{array}{c}\text { Ámbito } \\
\text { de conocimiento }\end{array}$ & Edad & $\begin{array}{l}\text { Disfrutó de beca } \\
\text { predoctoral }\end{array}$ & Categoría \\
\hline Profesora 1 & Experiment. & 41 & Sí & Agregada \\
\hline Profesora 2 & Experiment. & 36 & Sí & Agregada \\
\hline Profesora 3 & Sociales & 39 & Sí & Agregada \\
\hline Profesora 4 & Humanas & 39 & Sí & TU \\
\hline Profesora 5 & Sociales & 41 & Sí & TU \\
\hline Profesora 6 & Humanas & 34 & No & TU \\
\hline Profesora 7 & Tecnológicas & 35 & No & TU \\
\hline Profesora 8 & Salud & 38 & No & TU \\
\hline Profesora 9 & Salud & 37 & Sí & TU \\
\hline Profesora 10 & Tecnológicas & 38 & Sí & TU \\
\hline Profesora 11 & Sociales & 37 & No & TU \\
\hline Profesor 1 & Experiment. & 37 & Sí & TU \\
\hline Profesor 2 & Tecnológicas & 36 & Sí & Agregado \\
\hline Profesor 3 & Salud & 35 & Sí & Agregado \\
\hline Profesor 4 & Experiment. & 36 & Sí & Agregado \\
\hline Profesor 5 & Sociales & 38 & Sí & TU \\
\hline Profesor 6 & Salud & 38 & Sí & TU \\
\hline Profesor 7 & Tecnológicas & 43 & Sí & Agregado \\
\hline Profesor 8 & Humanas & & Sí & TU \\
\hline Profesor 9 & Sociales & 38 & No & TU \\
\hline Profesor 10 & Sociales & 38 & Sí & TU \\
\hline Profesor_11 & Experiment. & 37 & Sí & TU \\
\hline
\end{tabular}




\section{REFERENCIAS BiBLIOGRÁFICAS}

Barnes, B. 2007. "Catching up with Robert Merton: scientific collectives as status groups." Journal of Classical Sociology 7: 179-192.

Barnes, B. y R. G. A. Dolby. 1970. "The scientific ethos: a deviant viewpoint." Archive of European Sociology 11:3-25

Braun, D. 1998. "The role of funding agencies in the cognitive development of science." Research Policy 27: $807-821$.

Boudon, R. 1998. "Social mechanisms without black boxes." Pp. 172-203 in Social mechanisms. An analytical approach to social theory, edited by Hedström, P. \& R. Swedberg. Cambridge: Cambridge University Press.

Boudon, R. 2004. "La sociología que realmente importa." Papers 72: 215-226.

Browne, I. and P. England. 1997. "Oppression from within and without in sociological theories: an application to gender." Current Perspectives in Social Theory 17: 77-104.

Clark, J. 2005. "Women and science: leaky pipeline or gender filter?." Gender and education 17: 369386.

Correll, S. J. 2004. "Constraints into preferences: gender, status, and emerging career aspirations." American Sociological Review 69: 93-113.

Cruz-Castro, L., L. Sanz-Menéndez y J. Aja. 2006. Las trayectorias profesionales y académicas de los profesores de universidad y los investigadores del CSIC. Madrid: Consejo Superior de Investigaciones Científicas.

Eiduson, B. T. 1962. Scientists: Their psychological world. New York: Basic Books.

Elster, J. 1988. Uvas amargas: sobre la subversión de la racionalidad. Barcelona: Edicions 62.

Elster, J. 1989. Tuercas y tornillos. Una introducción a los conceptos básicos de las ciencias sociales. Barcelona: Gedisa.

Elster, J. 2007. Explainig social behaviour. More nuts and bolts for the social sciences. Cambridge: Cambridge University Press.

European Commission .2004. Gender and excellence in the making. Bruselas: Directorate-General for Research Information and Communication Unit . Consultado el 15 de mayo de 2007. (http:// ec.europa.eu/research/science-society/pdf/bias_brochure_final_en.pdf).

European Commission. 2005. Women and Science. Excellence and Innovation - Gender Equality in Science. Bruselas: Directorate-General for Research Information and Communication Unit. Consultado el 15 de mayo de 2007. (http://ec.europa.eu/research/science-society/pdf/documents women_sec_en.pdf) 
Fernández-Carro, R. 2009. "La teoría principal-agente en los estudios de ciencia y tecnología." Arbor. Ciencia, Pensamiento y cultura 738: 809-824.

Fernández, M. 2002. La formación de investigadores científicos en España. Madrid: CIS-Siglo XXI.

Fernández Esquinas, M. y C. Torres. 2009. "La ciencia como institución social: clásicos y modernos institucionalismos en la sociología de la ciencia." Arbor. Ciencia, Pensamiento y cultura 738: 663-687.

Fox, M. F. 1995. "Woman and Scientific Careers." Pp. 205-224 in Jasanoff, S. et al. (eds). Handbook of Science and Technology Studies. London: Sage.

Fox, M. y P. E. Stephan. 2001. "Careers of young scientists. Gender." Social Estudies of Science 31:109-122.

Funtowicz, S. O., y Ravetz, J. R. 1993. "Science for the Post-Normal Age." Futures 25 (7): 735-755

González, M. T., M. Pérez y M. Fernández. 1995. Trayectorias ocupacionales de los becarios de investigación españoles (1982-1992). Madrid: Consejo Superior de Investigaciones Científicas. Instituto de Estudios Sociales Avanzados.

Hedström, P. y R. Swedberg. 1996. "Social Mechanisms." Acta Sociologica 39: 281-308.

Hedström, P. y R. Swedberg (eds.). 1998. Social Mechanisms. An Analytical Approach to Social Theory. Cambridge: Cambridge University Press.

Huff, T. E. 2007. "Some historical roots of the ethos of science." Journal of Classical Sociology 7: 193210.

Izquierdo, M. J. 1998. El malestar en la desigualdad. Madrid: Catedra.

Izquierdo, M. J. (Dir.), E. Mora, L. Duarte i F. J. León. 2004. El sexisme a la UAB. Propostes d'actuació i dades per a un diagnòstic. Barcelona: Bellaterra, Servei de Publicacions de la Universitat Autònoma de Barcelona.

Izquierdo, M. J., F. J. León, H. Akhrif, L. Duarte y B. Martí. 2008. Foros y talleres de trabajo del I Congreso Internacional sobre sesgo de género y desigualdades en la evaluación de la calidad académica. Barcelona: UAB-Observatori per a la Igualtat, Bellaterra.

Kalleberg, R. 2007. "A reconstruction of the ethos of science." Journal of Classical Sociology 7: 137-160.

Leydesdorff, H. y H. Etzkowitz. 1996. "Emergence of a Triple Helix of University-Industry-Government Relations." Science and Public Policy 23: 279-286.

McClelland, D. 1979. "On the dynamics of creative physical scientists." Pp. 309-341 in Hudson, L. (ed.). The Ecology of Human Intelligence, England: Penguin.

Merton, R. K. 1948. Teoría y estructura sociales. México: FCE.

Merton, R. K. 1977a. "La estructura normativa de la ciencia." Pp. 355-362 en La sociología de la ciencia. vol 2. Madrid: Alianza Universidad. 
Merton, R. K. 1977b. "Las prioridades en los descubrimientos científicos." Pp. 377-422 en La sociología de la ciencia. vol 2. Madrid: Alianza Universidad.

Mitroff, I. 1974. "The Apollo Moon Scientist:A case study of the ambivalence of scientists." American Sociological Review 39: 579-595.

Roe, A. 1953. The making of a scientist. New York: Dodd, Mead.

Rothman, M. 1972. "A dissenting view on the scientific ethos." The British Journal of Sociology 23: 102-108.

Scott, J. y M. F. Fox. 1995. "Scientific careers: universalism and particularism." Annual Review of Sociology 21: 45-71.

Slaughter, S. y L. L. Leslie. 1997. Academic Capitalism: Politics, Policies and the Entrepreneurial University. Baltimore: Johns Hopkins University Press.

Turner, S. 2007. "Merton's 'Norms' in political and intellectual context." Journal of Classical Sociology 7: $161-178$.

Wyer, M. 1997. "Women, the doctorate, and equity in engineering education." Pp. 82-91 in International Symposium on Technology and Society, June 20-21, Glasgow :IEEE.

Ziman, J. 1996. "'Postacademic science':constructing knowledge with networks and norms." Science Studies 9: 67-80.

FRANCISCO JOSÉ LEÓN MEDINA es Doctor en Sociología. Profesor asociado de la Universidad Autónoma de Barcelona y de la Universidad de Girona. Ha sido miembro del GESES (Grupo de Estudios Emociones, Sentimientos y Sociedad) e investigador del Observatorio para la Igualdad de la UAB. En la actualidad, es investigador del GSADI (Grupo de Sociología Analítica y Diseño Institucional, de la UAB). Ámbito de investigación: emergencia de normas sociales, acción colectiva y bienes publicos, simulación social.

ENRICO MORA MALO es Doctor en Sociología y, actualmente, profesor agregado de la Universitat Autònoma de Barcelona, e investigador permanente del grupo de investigación Grup d'Estudis Sentiments, Emocions i Societat (GESES) del Departamento de Sociología de la Universitat Autònoma de Barcelona. Ámbito de investigación: Las desigualdades sociales según las relaciones de género y de clase, tanto bajo relaciones mercantiles como domésticas, teniendo en cuenta el impacto de dichas relaciones en la formación de las subjetividades y de las emociones, y en las prácticas de cuidado y provisión.

\section{RECIBIDO: $19 / 06 / 08$}

ACEPTADO:02/03/10

Publicado on-line: 22 de abril 2010 